\title{
INEQUALITIES FOR EIGENVALUES OF SUPPORTED AND FREE PLATES*
}

\author{
BY \\ L. E. PAYNE \\ University of Maryland
}

1. Introduction. Much attention has been given in the literature to the classical vibration and buckling problems for a plate if the boundary is clamped (see for instance Weinstein [15], Aronszajn [1], Aronszajn and Donoghue [2], Polya and Szegö [11], Payne [9], and others). Many of the methods which have been proposed for handling such problems are not practical if the boundaries of the plate are supported, free, or satisfy mixed conditions. Of course one has a minimum principle for computing upper bounds for the eigenvalues, but in applications lower bounds are usually more important.

In this report we establish certain "isoperimetric" inequalities for the eigenvalues in the classical vibration and buckling problems for supported and free plates. These inequalities involve the geometry of the plate and the eigenvalues of membranes having the same shape as that of the plate.

It is well known that if the boundary of a supported plate is rectilinear the eigenvalues in the vibration and buckling problems are related to those of the corresponding fixed membrane problem. If the boundary is not rectilinear this is no longer the case, and to my knowledge no relationship between the eigenvalues of the supported plate and those of the membrane is then known. We shall prove in this report that if the boundary is convex the eigenvalues of the supported plate are never greater than the corresponding eigenvalues of the fixed membrane of the same shape.

Let us suppose that the plate occupies a region $D$ bounded by a closed curve $C$ in the $x y$ plane. In comparing the eigenvalues of the plate problem to those of the membrane problem we assume the latter problem to be defined over the same region. We consider the following eigenvalue problems:

I.

$$
\Delta u+\lambda u=0 \quad \text { in } \quad D, \quad u=0 \text { on } C\left(\Delta=\frac{\partial^{2}}{\partial x^{2}}+\frac{\partial^{2}}{\partial y^{2}}\right)
$$

II.

$$
\Delta v+\mu v=0 \quad \text { in } \quad D, \quad \frac{\partial v}{\partial \nu}=0 \text { on } C
$$

III.

$$
\Delta^{2} w+\Lambda \Delta w=0 \quad \text { in } D, \quad w=0, \quad M(w)=0 \quad \text { on } C
$$

IV.

$$
\Delta^{2} \varphi-\Omega^{2} \varphi=0 \text { in } D, \quad \varphi=0, \quad M(\varphi)=0 \text { on } C
$$

V.

$$
\Delta^{2} w+\Gamma \Delta w=0 \quad \text { in } \quad D, \quad M(w)=0, \quad V(w)=0 \quad \text { on } C
$$

VI.

$$
\Delta^{2} \varphi-\gamma^{2} \varphi=0 \quad \text { in } D, \quad M(\varphi)=0, \quad V(\varphi)=0 \text { on } C
$$

where $\nu$ denotes the outward normal on $C$ and $M(\psi)$ and $V(\psi)$ are defined as follows:

${ }^{*}$ Received December 10, 1956. This research was supported in part by the U. S. Air Force under Contract No. AF 18(600)-573-monitored by the Office of Scientific Research, Air Research and Development Command. 


$$
\begin{aligned}
& M(\psi)=\sigma \Delta \psi+(1-\sigma)\left[\frac{\partial}{\partial \nu}\left(\frac{\partial \psi}{\partial x}\right) \frac{\partial x}{\partial \nu}+\frac{\partial}{\partial \nu}\left(\frac{\partial \psi}{\partial y}\right) \frac{\partial y}{\partial \nu}\right], \quad 0<\sigma<\frac{1}{2} \\
& V(\psi)=\frac{\partial}{\partial \nu}(\Delta \psi)-(1-\sigma) \frac{\partial}{\partial s}\left[\frac{\partial}{\partial \nu}\left(\frac{\partial \psi}{\partial y}\right) \frac{\partial x}{\partial \nu}-\frac{\partial}{\partial \nu}\left(\frac{\partial \psi}{\partial x}\right) \frac{\partial y}{\partial \nu}\right] .
\end{aligned}
$$

As is well known, I and II characterize the fixed and free membrane problems respectively. The buckling problem and vibration problem for a supported plate are given by III and IV, while V and VI are the corresponding problems for a free plate.

In each of these problems it is well known that non-trivial solutions exist if and only if the constant $\lambda, \mu, \Lambda, \Omega, \Gamma$ and $\gamma$ take on certain discrete non-negative values. We order these eigenvalues as

$$
\lambda_{n} \geq \lambda_{m}, \quad n>m,
$$

the other eigenvalue being similarly ordered. We denote the eigenfunction corresponding to $\lambda_{n}$ by $u_{n}$, etc.

In this paper we establish the following isoperimetric inequalities for $C$ convex:

$$
\begin{aligned}
& \Lambda_{n} \leq \lambda_{n}, \\
& \Omega_{n} \leq \lambda_{n}, \\
& \Gamma_{n} \leq \mu_{n}, \\
& \gamma_{n} \leq \mu_{n} .
\end{aligned}
$$

For arbitrary $C$ we prove further that

$$
\begin{gathered}
\Lambda_{n} \geq(1-\sigma) \mu_{2}+\sigma \lambda_{n}, \\
\Lambda_{n} \geq\left(\frac{1+\sigma}{2}\right) \lambda_{n}, \\
\Lambda_{n} \geq \Gamma_{n+3} \geq(1-\sigma) \mu_{|(n+3) / 2|}, \\
\Omega_{n}^{2} \geq \lambda_{n} \Lambda_{1}, \\
\gamma_{n+2}^{2} \geq \mu_{n} \Gamma_{4} \geq(1-\sigma) \mu_{2} \mu_{n},
\end{gathered}
$$

where the symbol $|(n+3) / 2|$ is used to denote the largest integer contained in the quantity $(n+3) / 2$.

We first define

$$
\mathfrak{A}(\psi)=(1-\sigma)\left[D\left(\frac{\partial \psi}{\partial x}\right)+D\left(\frac{\partial \psi}{\partial y}\right)\right]+\sigma \iint_{D}(\Delta \psi)^{2} d A .
$$

The eigenvalues in each of the plate problems which we consider are defined as the minimum value assumed by $\mathfrak{A}(\psi)$ for a certain class of continuously differentiable functions $\psi$. In (12) $D$ denotes the Dirichlet integral, i.e.

$$
D(\psi)=\iint_{D}|\operatorname{grad} \psi|^{2} d A
$$


2. Buckling problem for the plate. A. Supported boundary. The eigenvalues $\Lambda_{n}$ in problem III are defined by the following minimum principle:

$$
\Lambda_{n}=\min . \mathfrak{A}(\psi)
$$

among all continuously differentiable functions which vanish on $C$ and satisfy the relations

$$
D\left(\psi, w_{i}\right)=\iint_{D}\left[\frac{\partial \psi}{\partial x} \frac{\partial w_{i}}{\partial x}+\frac{\partial \psi}{\partial y} \frac{\partial w_{i}}{\partial y}\right] d A=0, \quad i=1,2, \cdots, n-1,
$$

and

$$
D(\psi)=1
$$

We note that if $C$ is convex its radius of curvature $\rho$ is everywhere positive. Likewise if we denote by $r$ the distance from an arbitrary origin inside a convex curve $C$ then the quantity $h=r(\partial r / \partial \nu)$ is positive at every point on $C$.

We assume first that the region is convex and choose for $\psi$,

$$
\psi=\sum_{i=1}^{n} a_{i} u_{i},
$$

where the functions $u_{i}$ are the eigenfunctions of the corresponding fixed membrane problem (I) and the $a_{i}$ are so determined as to satisfy (14) and (15). Insertion of (16) in (13) gives after an application of Green's formula,

$$
\Lambda_{n} \leq \sum_{i=1}^{n} a_{i}^{2} \lambda_{i} D\left(u_{i}\right)+\frac{(1-\sigma)}{2} \oint_{c} \frac{\partial}{\partial \nu}\left[\left(\sum_{i=1}^{n} a_{i} \frac{\partial u_{i}}{\partial x}\right)^{2}+\left(\sum_{i=1}^{n} a_{i} \frac{\partial u_{i}}{\partial y}\right)^{2}\right] d s .
$$

We have used the fact that

$$
D\left(u_{i}, u_{i}\right)=0, \quad i \neq j .
$$

Now the boundary integral in (17) may be rewritten as

$$
\begin{aligned}
\oint_{c} \frac{\partial}{\partial \nu}\left[\left(\sum_{i=1}^{n} a_{i} \frac{\partial u_{i}}{\partial x}\right)^{2}+\left(\sum_{i=1}^{n} a_{i} \frac{\partial u_{i}}{\partial y}\right)^{2}\right] d s & =\oint_{c} \sum_{i=1}^{n} a_{i} \frac{\partial u_{i}}{\partial \nu} \sum_{i=1}^{n} a_{i} \Delta u_{i} d s \\
& +\oint_{c}\left(\sum_{i=1}^{n} a_{i} \frac{\partial u_{i}}{\partial y}\right)^{2} \frac{\partial}{\partial s}\left(\frac{\sum_{i=1}^{n} a_{i} \frac{\partial u_{i}}{\partial x}}{\sum_{i=1}^{n} a_{i} \frac{\partial u_{i}}{\partial y}}\right) d s,
\end{aligned}
$$

where $\partial / \partial s$ denotes differentiation along the curve $C$. The first boundary integral on the right vanishes if the region is convex since the $u_{i}$ satisfy $I$. We now make use of the fact that on $C$

$$
\frac{\partial \psi}{\partial s}=\sum_{i=1}^{n} a_{i} \frac{\partial u_{i}}{\partial x} \frac{\partial x}{\partial s}+\sum_{i=1}^{n} a_{i} \frac{\partial u_{i}}{\partial y} \frac{\partial y}{\partial s}=0,
$$

and find finally that

$$
\oint_{c} \frac{\partial}{\partial \nu}\left[\left(\sum_{i=1}^{n} a_{i} \frac{\partial u_{i}}{\partial x}\right)^{2}+\left(\sum_{i=1}^{n} a_{i} \frac{\partial u_{i}}{\partial y}\right)^{2}\right] d s=-\oint_{c} \frac{1}{\rho}\left(\sum_{i=1}^{n} a_{i} \frac{\partial u_{i}}{\partial \nu}\right)^{2} d s \leq 0 .
$$


Thus from (17)

$$
\Lambda_{n} \leq \sum_{i=1}^{n} a_{i}^{2} \lambda_{i} D\left(u_{i}\right) \leq \lambda_{n} \sum_{i=1}^{n} a_{i}^{2} D\left(u_{i}\right)=\lambda_{n},
$$

the last step following from the normalization (15). This establishes inequality (3). Note that we may obtain a better inequality for the first eigenvalue $\Lambda_{1}$, for in this case we have only one eigenfunction $u_{1}$ in (16). The quantity on the right hand side of (21) can then be approximated. Let us suppose that the function is normalized according to (15) (then $a_{1}=1, a_{i}=0, i>1$ ). According to Rellich's identity [12],

$$
1=\lambda_{1} \iint_{D} u_{1}^{2} d A=\frac{1}{2} \oint_{C} h\left(\frac{\partial u_{i}}{\partial \nu}\right)^{2} d s,
$$

where $h=r(\partial r / \partial \nu)$ and the origin is arbitrary. We choose the origin so as to minimize the quantity $h_{\max }$ on $C$ and denote by $h^{0}$ the resulting $h_{\max }$. We obtain then the inequality,

$$
\Lambda_{1} \leq \lambda_{1}-\frac{2(1-\sigma)}{h^{0} \rho_{\max }}
$$

As was previously mentioned the equality sign holds in (22) and (24) if the boundary $C$ is rectilinear $\left(\rho_{\max }=\infty\right)$. It is easily seen from (21) that this is the only case in which equality exists.

Inequality (7) for a general plate (not necessarily convex) follows directly from the maximum-minimum principle for $\Lambda_{n}$ (see Courant-Hilbert [3]) or from the second monotony principle (see Aronszajn and Donoghue [2]). One need merely observe that in problem II, $\mu_{1}=0\left(v_{1}=\right.$ constant $)$ and $\mu_{2}$ is defined as

$$
\mu_{2}=\min \cdot \frac{D(\chi)}{\iint_{D} \chi^{2} d A},
$$

for continuous functions $\chi$ which satisfy the condition

$$
\iint_{D} x d A=0
$$

It follows then that, since the functions $\psi$ in (13) vanish on $C$,

$$
\iint_{D} \frac{\partial \psi}{\partial x} d A=\iint_{D} \frac{\partial \psi}{\partial y} d A=0
$$

Hence from (25)

$$
D\left(\frac{\partial \psi}{\partial x}\right) \geq \mu_{2} \iint_{D}\left(\frac{\partial \psi}{\partial x}\right)^{2} d A ; \quad D\left(\frac{\partial \psi}{\partial y}\right) \geq \mu_{2} \iint_{D}\left(\frac{\partial \psi}{\partial y}\right)^{2} d A .
$$

Inequality (7) then follows directly from the maximum-minimum principle.

Inequality (8) is established by making use of the minimum principle for $\lambda_{n}$, i.e. 


$$
\lambda_{n}=\min \cdot \frac{D(\chi)}{\iint_{D} \chi^{2} d A}
$$

for continuous functions $\chi$ which vanish on $C$ and satisfy the condition

$$
\iint_{D} \chi u_{i} d A=0, \quad i=1,2, \cdots, n-1 .
$$

By Green's theorem and Schwarz's inequality we have

$$
\frac{D(\chi)}{\iint_{D} \chi^{2} d A} \leq \frac{\iint_{D}(\Delta \chi)^{2} d A}{D(\chi)} .
$$

Thus,

$$
\begin{aligned}
& \iint_{D}(\Delta \chi)^{2} d A \\
\lambda_{n} & \leq \frac{\int(\chi)}{D(\chi)}=\frac{\int\left(\frac{\partial^{2} \chi}{\partial x^{2}} \frac{\partial^{2} \chi}{\partial y^{2}}-\left(\frac{\partial^{2} \chi}{\partial x \partial y}\right)^{2}\right] d A}{D(\chi)}+2(1-\sigma) \frac{\iint_{D}}{\iint_{D}(\Delta x)^{2} d A} \\
& \leq \frac{\mathfrak{A}(\chi)}{D(\chi)}+\frac{(1-\sigma)}{2} \frac{(\chi)}{D} \\
& \leq\left[1+\left(\frac{1-\sigma}{2}\right)^{2}+\left(\frac{1-\sigma}{2}\right)^{2}+\cdots+\left(\frac{1-\sigma}{2}\right)^{\rho}+\cdots\right] \frac{\mathfrak{A}(\chi)}{D(\chi)}
\end{aligned}
$$

Thus we obtain for arbitrary $x$

$$
\lambda_{n} \leq \frac{2}{1+\sigma} \frac{\mathfrak{M}(\chi)}{D(\chi)} .
$$

We now choose $\chi$ to be a linear combination of the first $n$ eigenfunctions $w_{i}$ of problem III with the constants chosen to satisfy (30) and inequality (8) follows immediately.

B. Free boundary. The eigenvalues $\Gamma_{n}$ of problem $V$ are defined by Eqs. (13), (14), and (15). However, we now remove the restriction that $\psi$ vanish on the boundary. It is clear that the first three eigenvalues will be zero. The corresponding eigenfunctions are

$$
w_{1}=C_{1}, w_{2}=C_{2}+C_{3} x, w_{3}=C_{4}+C_{5} x+C_{6} y,
$$

where the $C_{i}$ are constants.

We assume first that the plate is convex, and choose instead of (16)

$$
\psi=\sum_{i=1}^{n} d_{i} v_{i} .
$$

Equation (17) follows with $u_{i}$ replaced by $v_{i}$ as does (19). We have instead of (20), since $\partial v_{i} / \partial \nu=0$ on $C$,

$$
\frac{\partial \psi}{\partial \nu}=\sum_{i=1}^{n} \alpha_{i} \frac{\partial v_{i}}{\partial x} \frac{\partial x}{\partial \nu}+\sum_{i=1}^{n} \alpha_{i} \frac{\partial v_{i}}{\partial y} \frac{\partial y}{\partial \nu}=0 .
$$


Instead of (21) we have in this case

$$
\oint_{c} \frac{\partial}{\partial \nu}\left[\left(\sum_{i=1}^{n} \alpha_{i} \frac{\partial v_{i}}{\partial x}\right)^{2}+\left(\sum_{i} \alpha_{i} \frac{\partial v_{i}}{\partial y}\right)^{2}\right] d s=-\oint_{c} \frac{1}{\rho}\left(\sum_{i=1}^{n} a_{i} \frac{\partial u_{i}}{\partial s}\right)^{2} d s \leq 0 .
$$

Thus from (17)

$$
\Gamma_{n} \leq \sum_{i=1}^{n} \alpha_{i}^{2} \mu_{i} D\left(v_{i}\right) \leq \mu_{n} \sum_{i=1}^{n} \alpha_{i}^{2} D\left(v_{i}\right)=\mu_{n} .
$$

This proves inequality (5).

For the general plate (not necessarily convex) we shall now establish (9). The left hand side of this inequality follows from the minimum principle for $\Gamma_{n+3}$ mentioned previously in this section. (The eigenvalues $\Gamma_{n}$ are defined by the right hand side of (13) under conditions (14) and (15). However, the trial functions $\psi$ are not required to vanish on $C$ ). We note that any function which vanishes on $C$ is orthogonal in Dirichlet norm to any harmonic function. (This is an immediate consequence of Green's identity). Hence, if we choose for $\psi$ functions which vanish on $C$, condition (14) is automatically satisfied for the first three eigenfunctions (34). In particular let

$$
\psi=\sum_{i=1}^{n} b_{i} w_{i}^{*},
$$

where the upper index $s$ indicates that we are using the eigenvalues of the supported plate. The $b_{i}$ are chosen to satisfy (14) and (15). Then

$$
\Gamma_{n+3} \leq \mathfrak{A}(\psi)=\sum_{i=1}^{n} b_{i}^{2} \mathfrak{H}\left(w_{i}^{s}\right)=\sum_{i=1}^{n} b_{i}^{2} \Lambda_{i} D\left(w_{i}^{s}\right) \leq \Lambda_{n} .
$$

In order to establish the right hand side of (9) we recall the definition for $\mu_{n}$, i.e.

$$
\mu_{n}=\min \cdot \frac{D(\chi)}{\iint_{D} \chi^{2} d A}
$$

for continuous functions $\chi$ which satisfy the condition

$$
\iint_{D} \chi v_{i} d A=0, \quad i=1,2, \cdots, n-1 .
$$

We choose for $\chi$ two functions

$$
\chi_{1}=\sum_{i=2}^{2 n} \beta_{i} \frac{\partial w_{i}}{\partial x}, \chi_{2}=\sum_{i=2}^{2 n} \beta_{i} \frac{\partial w_{i}}{\partial y}
$$

with the $\beta_{i}$ chosen to satisfy condition (42). The $w_{i}$ are the eigenfunctions of problem V. Then from (41)

$$
\mu_{n} \leq \frac{D\left(\chi_{1}\right)+D\left(\chi_{2}\right)}{\iint_{D}\left[\chi_{1}^{2}+\chi_{2}^{2}\right] d A} \leq \frac{D\left(\chi_{1}\right)+D\left(\chi_{2}\right)+\frac{\sigma}{1-\sigma} \iint_{D}\left[\frac{\partial \chi_{1}}{\partial x}+\frac{\partial \chi_{1}}{\partial y}\right]^{2} d A}{\iint_{D}\left[\chi_{1}^{2}+\chi_{2}^{2}\right] d A} .
$$


But since $\mathscr{Y}\left(w_{i}, w_{i}\right)=D\left(w_{i}, w_{i}\right)=0$ for $i \neq j$ this inequality reduces to

$$
\mu_{n} \leq \sum_{i=2}^{2 n} \frac{\beta_{i}^{2}\left[D\left(\frac{\partial w_{i}}{\partial x}\right)+D\left(\frac{\partial w_{i}}{\partial y}\right)+\frac{\sigma}{1-\sigma} \iint_{D}\left(\Delta w_{i}\right)^{2} d A\right]}{\sum_{i=2}^{2 n} \beta_{i}^{2} D\left(w_{i}\right)} \leq \frac{1}{1-\sigma} \Gamma_{2 n} .
$$

We have then the inequality

$$
\Gamma_{2 n} \geq(1-\sigma) \mu_{n}
$$

or equivalently

$$
\Gamma_{n+3} \geq(1-\sigma) \mu_{|(n+3) / 2|}
$$

which was the relationship to be established.

3. Vibration problem for the plate. A. Supported boundary. The minimum principle for determining the eigenvalues $\Omega_{n}^{2}$ of problem IV is the following

$$
\Omega_{n}^{2}=\min . \mathfrak{A}(\psi)
$$

for continuously differentiable functions $\psi$ which vanish on $C$ and satisfy the conditions

$$
\iint_{D} \psi \varphi_{i} d A=0, \quad i=1,2, \cdots, n-1,
$$

and

$$
\iint_{D} \psi^{2} d A=1
$$

Let us assume for the moment that $C$ is convex. Proceeding exactly as we did in establishing (3) we prove with little difficulty that

$$
\Omega_{n}^{2} \leq \lambda_{n}^{2} \text {. }
$$

Thus if we take $\Omega_{n}$ to be positive (4) follows immediately. One also obtains the improved bound for $\Omega_{1}^{2}$,

$$
\Omega_{1}^{2} \leq \lambda_{1}\left[\lambda_{1}-\frac{2(1-\sigma)}{h^{0} \rho_{\max }}\right] .
$$

We return again to the case of a general curve $C$. Inequality (10) follows directly from the minimum principle for $\lambda_{n},(29)$ and (30). We choose for $\psi$

$$
\psi=\sum_{i=1}^{n} c_{i} \varphi_{i}
$$

where the $c_{i}$ are chosen to satisfy (30). (We use the $\varphi_{i}$ of problem IV.) Then

$$
\lambda_{n} \leq \frac{\mathfrak{R}(\psi)}{\iint_{D} \psi^{2} d A} \frac{D(\psi)}{\mathfrak{R}(\psi)} \leq \frac{\sum_{i=1}^{n} c_{i}^{2} \Omega_{i}^{2} \iint_{D} \varphi_{i}^{2} d A}{\sum_{i=1}^{n} c_{i}^{2} \iint_{D} \varphi_{i}^{2} d A} \cdot \frac{1}{\Lambda_{1}} \leq \Omega_{n}^{2} / \Lambda_{1}
$$


which is (10). In a similar way we could have obtained the inequality,

$$
\Omega_{n}^{2} \geq \Lambda_{n-m} \lambda_{m+1}, \quad 0 \leq m \leq n-1 .
$$

From (55) and (8) we obtain at once

$$
\Omega_{n}^{2} \geq \frac{(1+\sigma)}{2} \lambda_{n-m} \lambda_{m+1}, \quad 0 \leq m \leq n-1 .
$$

B. Free boundary. We turn now to problem VI. The minimum principle for $\gamma_{n}^{2}$ is given by

$$
\gamma_{n}^{2}=\min . \mathfrak{A}(\psi)
$$

among all continuously differentiable functions $\psi$ which satisfy conditions (49) and (50). As in the case of the buckling problem for the free plate, the first three eigenvalues are zero with corresponding eigenfunctions

$$
\begin{aligned}
& \varphi_{1}=k_{1} \\
& \varphi_{2}=k_{2}+k_{3} x \\
& \varphi_{3}=k_{4}+k_{5} x+k_{6} y, \quad k_{i}=\text { constant. }
\end{aligned}
$$

For convex $C$ the proof of inequality (6) follows along the same lines as the proof of (5). Hence we shall not repeat the details of the proof.

For general $C$ the first half of inequality (11) is proved in the same manner as was (10). The second half then follows from (9). To prove (11) we make use of the minimum principle for $\mu_{n}$ given by (41) and (42). We may rewrite (41) as

$$
\mu_{n} \leq \frac{\mathfrak{A}(\chi)}{\iint_{D} \chi^{2} d A} \frac{D(\chi)}{\mathfrak{A}(\chi)},
$$

and choose for $\chi$

$$
\chi=\sum_{i=1}^{n+2} \delta_{i} \varphi_{i},
$$

where the $\varphi_{i}$ are the eigenvalues of problem VI. The $\delta_{i}$ are chosen to satisfy (42), and in addition the conditions

$$
D\left(w_{2}, \chi\right)=D\left(w_{3}, \chi\right)=0, \quad D(\chi)=1,
$$

where $w_{2}$ and $w_{3}$ are given by (34). [The condition $D\left(w_{1}, \chi\right)=0$ is satisfied identically.] Then

$$
\begin{aligned}
\mu_{n} & \leq \frac{\sum_{i=1}^{n+2} \delta_{i}^{2} \gamma_{i}^{2} \iint_{D} w_{i}^{2} d A}{\sum_{i=1}^{n+2} \delta_{i}^{2} \iint_{D} w_{i}^{2} d A} \cdot \frac{1}{\Gamma_{4}} \\
& \leq \gamma_{n+2}^{2} / \Gamma_{4}
\end{aligned}
$$


which is the first half of (11). In a similar way we establish the inequality

$$
\gamma_{n+3}^{2} \geq \mu_{2+m} \Gamma_{n-m+3}, \quad 0 \leq m \leq n-1 .
$$

With the help of (49) this inequality gives

$$
\gamma_{n+3}^{2} \geq(1-\sigma) \mu_{2+m} \mu_{|(n-m+3) / 2|}, \quad 0 \leq m \leq n-1 .
$$

The right hand side is a maximum if $m=n-1$. We obtain then

$$
\gamma_{n+3}^{2} \geq(1-\sigma) \mu_{2} \mu_{n+1} \text {. }
$$

4. Concluding remarks. The eigenvalues of the vibration and buckling problems for a supported or free plate have been bounded in terms of the eigenvalues of the corresponding fixed or free membrane problems. If these cannot be determined explicitly they are still more easily bounded than the eigenvalues of the plate problems which we have considered. For instance the eigenvalues of the fixed membrane are monotone with region while those in the plate problems which we have considered are not. Under certain symmetry conditions one can obtain a lower bound for the first non-zero eigenvalue in the free membrane problem which involves only the geometry of the region (see Payne and Weinberger [10]). We have also the isoperimetric inequalities

$$
\begin{aligned}
& \lambda_{1} \geq \frac{\pi \gamma^{2}}{A}, \quad j \approx 2.4048, \\
& \mu_{2} \leq \frac{\pi p^{2}}{A}, \quad p \approx 1.8412,
\end{aligned}
$$

where $A$ is the area of $D$. The first inequality was proved independently by G. Faber [4], and E. Krahn [7], and the second was formulated by Kornhauser and Stakgold [6], proved under certain restrictive conditions by G. Szegö [13] and established for a general region by Weinberger [14]. From (66), (8) and (56) we have the inequalities

$$
\Lambda_{1} \geq \frac{(1+\sigma)}{2} \frac{\pi j^{2}}{A}, \Omega_{1} \geq\left[\frac{(1+\sigma)}{2}\right]^{1 / 2} \frac{\pi j^{2}}{A} .
$$

We note further that inequalities (3) to (10) hold in any number of dimensions. The right hand side of (9) must merely be changed to read

$$
\Gamma_{N(n-1)+2} \geq(1-\sigma) \mu_{n},
$$

where $N$ is the number of dimensions.

In $N$ dimensions inequality (11) is replaced by

$$
\gamma_{n+N}^{2} \geq \mu_{n} \Gamma_{N+2} \geq(1-\sigma) \mu_{2} \mu_{n} .
$$

In case of a vibrating free plate in the shape of a square, Nakata and Fujita [8] have applied the method of Kato [5] and after considerable computational work have obtained both upper and lower bounds for the first non-zero eigenvalue. For $\sigma=0.225$ and a square of side 2 they obtained the bounds

$$
3.418<\gamma_{4}<3.554 \text {. }
$$

However, their method is not completely rigorous. If we make use of the symmetry of 
the region' we obtain the bounds

$$
3.06<\gamma_{4}<4.94 \text {. }
$$

The upper bound is, of course, not good and a close bound can easily be obtained anyway by the usual Rayleigh-Ritz procedure. However, one finds immediately, with no computational work whatsoever, the lower bound given in (71) which in application may be quite good enough.

\section{BibLIOGRAPHY}

1. N. Aronszajn, Studies in eigenvalue problems, Tech. Rep. 3, Oklahoma A. and M. College (1951)

2. N. Aronszajn and W. Donoghue, Studies in eigenvalue problems, Tech. Rep. 12, University of Kansas (1954)

3. R. Courant and D. Hilbert, Methoden der mathematischen Physik, vol. 1, Berlin, 1931

4. G. Faber, Beweis, dass unter allen homogenen Membranen von gleicher Flache und gleicher Spannung die kreisförmige den tiefsten Grundton gibt, Sitz. ber. Bayr. Akad, Wiss., 169 (1923)

5. T. Kato, On some approximation methods concerning the operator $T^{*} T$, Math. Ann. 126, 253 (1953)

6. E. T. Kornhauser and T. Stakgold, $A$ variational theorem for $\nabla^{2} u+\lambda u=0$ and its applications, J. Math. Phys. 31, 45 (1952)

7. E. Krahn, Über eine von Rayleigh formulierte Minimaleigenschaft des Kreises, Math. Ann. 94, 97 (1924)

8. Y. Nakata and H. Fujita, On upper and lower bounds of the eigenvalues of a free plate, J. Phys. Soc. Japan, 10, 823 (1955)

9. L. E. Payne, Inequalities for eigenvalues of membranes and plates, J. Ratl. Mech. Anal. 4, 517 (1955)

10. L. E. Payne and H. F. Weinberger, Two inequalities for eigenvalues of membranes, Tech. Note BN-65, Univ. of Maryland (1955)

11. G. Polya and G. Szegö, Isoperimetric inequalities in mathematical physics, Ann. Math. Studies 27, Princeton (1951)

12. F. Rellich, Darstellung der Eigenwerte von $\Delta u+\lambda u$ durch ein Randintegral, Math. Z. 46, 635 (1940)

13. G. Szegö, Inequalities for certain eigenvalues of a membrane of given area, J. Ratl. Mech. Anal. 3, 343 (1954)

14. H. F. Weinberger, $A n$ isoperimetric inequality for the free membrane problem, J. Ratl. Mech. Anal. $5,633(1956)$

15. A. Weinstein, Elude des spectres des équations aux dérivées partielles de la théorie des plaques élastiques, Mémorial de Sciences Math. 88, Paris (1937)

\footnotetext{
${ }^{1}$ In the case of a square plate it can be easily shown that inequality (62) may be replaced by: $\boldsymbol{r}^{2}{ }^{2} \geq \mu_{\boldsymbol{m}} \Gamma_{4}$.
} 